\title{
Seasonal changes in endogenous hormone and sugar contents during bud dormancy in tree peony
}

\author{
Philip M.P. Mornya ${ }^{1,2 *}$ and Fangyun Cheng ${ }^{1}$ \\ ${ }^{1}$ Center for National Flower Engineering Research, College of Landscape Architecture, Beijing Forestry University, \\ Beijing-100083, China. ${ }^{2}$ School of Forestry and Horticulture, Njala University, Sierra Leone, West Africa. \\ *E-mail: philip_mornya@yahoo.com
}

\begin{abstract}
The trial to investigate hormonal and sugar changes in tree peony buds associated with dormancy was conducted in the field at the Beijing Forestry University Experimental Site in China during autumn, winter and spring seasons (2009/2010 and 2010/2011), the periods of dormancy development and release. The experimental design was randomized complete block with three replications. The hormone and sugar levels were determined using the enzyme-linked immunosorbent assay (ELISA) technique and spectrophotometer, respectively. Winter temperature accumulated abscisic acid (ABA) and sugars in tree peony buds which most likely induced dormancy. Spring temperature, on the other hand, degraded ABA and sugars, and accumulated gibberellic acid $\left(\mathrm{GA}_{3}\right)$ that possibly released dormancy in tree peony buds indicating that environmental temperature was the key regulator of hormone and sugar levels that influenced bud dormancy and growth. The results suggest that accumulation of ABA, $\mathrm{GA}_{3}$ and sugars in buds during dormancy or bud-break stage appears to be directly related to the degree of temperature experienced at that stage. While ABA and sugar accumulated with decreasing temperature, $\mathrm{GA}_{3}$ accumulated with increasing temperature. It is likely that the reduction of $\mathrm{ABA}$ and sugars played an important role in bud dormancy release or alteration in bud growth of tree peonies. Seasonal patterns of sucrose and starch were almost the same in buds of the tested tree peony cultivars which contradicts previous studies suggesting converse relationship in terms of accumulation in winter. However, seasonal accumulation of endogenous compositions varies with cultivar. Among the investigated cultivars, 'Luoyang Hong' (LH) not only accumulated less ABA, GA 3 and sugars but also released bud dormancy earlier than the 'Zhao Fen' (ZF) and 'High Noon' (HN), suggesting that the level of these internal compositions in LH is less responsive to seasonal temperature change. The ability of buds to simultaneously accumulate ABA and sugar reserves while in dormant state may provide a significant adaptive advantage for peonies to survive the erratic climate, particularly in temperate regions, which could be one of the reasons for the geographically widespread of the genus Paeonia in the world.
\end{abstract}

Keywords: Bud dormancy, carbohydrates, plant hormone, temperature, tree peony

\section{Introduction}

An alternating short period of growth with long period of dormancy is a critical characteristic of the life cycle of tree peony plants (Paeonia Sect Moutan.). Shoot elongation hardly occurs during dormancy. The ability to adjust to seasonal temperature changes is essential for the survival of the plant. Especially in winter seasons in temperate regions where temperatures normally fall below $0{ }^{\circ} \mathrm{C}$, cold hardiness of woody plants is critical (Burke et al., 1976). In order to survive, plants in such regions must stop growth in autumn, well in advance of the onset of cold temperatures. With the cessation of growth and initiation of dormancy, radical adjustments needed for survival begin in plants (Weiser, 1970). This period of dormancy and non-growth is therefore an adaptive response of temperate woody plants to seasonal changes in temperature. Growth pattern adapts with the environment, which subsequently prepares the plants to endure cold winter conditions.

Tree peony buds normally sprout, grow and blossom in spring. Flower organ differentiation and development occur in early summer to autumn followed by leaf shedding and bud hardening in cold winter seasons. Generally, tree peony buds become dormant to survive the unfavorable conditions in late autumn to winter. Wang et al. (1998) noted that dormancy in tree peonies begins at the onset of low temperature and severe winter weather conditions, lasting through the next spring. Under both field and greenhouse conditions, dormancy release and bud growth into shoots and flowers during spring only occurs after exposure to sufficient cool temperatures (Byrne and Halevy, 1986; Wang et al., 1998). Temperature is therefore a critical element for dormancy initiation/release. But our understanding of these responses at physiological level is limited, particularly on the physiology underlying hormone and carbohydrate interactions with dormancy in tree peonies induced by temperatures.

The length of photoperiod greatly influences the cessation of growth and initiation of dormancy in woody plants (Olsen et al., 1997; Weiser, 1970). However, other factors such as hormones (Duan et al., 2004; Lavee and May, 1997), temperature (Junttila et al., 2003) and biotic and abiotic stresses (Chen and Li, 1978) also affect the timing and degree of dormancy. Different levels of endogenous abscisic acid (ABA) are associated with dormancy development and release in apical buds of plants (Powell, 1987; Qin et al., 2009). Rashid (2009) noted that high concentrations of ABA affect the translocation of amino acids and sugars in plants. Aside from ABA, gibberellins (GA) are also associated with dormancy of apical buds. GA generally accelerates plant 
growth. For example, Qin et al. (2009) noted that while growthpromoting hormones such as $\mathrm{GA}_{3}$ (gibberellic acid), IAA (indole3-acetic acid) and ZR (zeatin riboside) increase, growth-inhibiting hormones such as ABA decrease at dormancy release.

The levels of these hormones in the buds of plants are also mainly influenced by temperature. For instance, Pinthus et al. (1989) reported that increase in temperature increases endogenous $\mathrm{GA}_{1}$ in isogenic lines of Triticum. Interestingly, however, Thingnaes et al. (2003) observed that temperature did not affect the level of endogenous GA in Arabidopsis thaliana. Furthermore, Welling et al. (1997) noted that decreasing temperatures increase the levels of endogenous ABA in Betula pubescens. Thus, it is apparent that changes in temperature could affect hormonal levels in plants.

On the other hand, sucrose is noted to be associated with dormancy development and release in lily bulbs (Nowak et al., 1974). Report suggests that in plants, total soluble sugars increase at the onset of cold conditions, reach the maximum at full cold hardiness and decline during deacclimation (Sakai and Larcher, 1987). Stored sugar reserves are used in spring to provide energy for shoot growth and development (Otzen and Koridon, 1970).

These contrasting reports sometimes underscore the need for further research into the roles of hormones, sugars and temperature at bud dormancy and flower development in tree peonies. In commercial tree peony flower production, both under field and forcing conditions, bud dormancy and germination are crucial processes that determine flowering time and flower quality. Therefore, understanding how changes in temperature influence the levels of hormones and sugars along with the timing and degree of dormancy and the related processes is important. The objective of this study was to determine the changes in hormonal and sugar levels in buds associated with dormancy during autumn, winter and spring seasons in tree peony plants. The findings of the study would not only explain the basis for physiological changes at dormancy of peonies, but would also help to improve on the protocol for flower forcing in tree peony industry.

\section{Materials and methods}

Plant sample collection: Eight-year old grafted 'Louyang Hong' (LH), 'Zhao Fen' (ZF) and 'High Noon' (HN) tree peony ( $P$. Sect Moutan.) cultivars with similar growth vigor, field-grown at Beijing Forestry University experimental site in China were selected for the investigation. The study was conducted for two consecutive tree peony cropping seasons of 2009 and 2010. For both trials, the plants were selected in late summer during which time the shoots still largely retained their leaves and flower bud differentiation was in progress.

Samples were collected before leaf-fall in autumn (late September to mid October); during chilling accumulation in winter (mid December to mid January) and after chilling condition in spring (March). Specifically sample collection for both trials started in autumn (29 $9^{\text {th }}$ September and $28^{\text {th }}$ September), winter $\left(17^{\text {th }}\right.$ December and $16^{\text {th }}$ December) of 2009 and 2010 cropping seasons. Samples were collected three times during each sampling period at 15-day intervals with at least 10 apical buds randomly collected each time as sample materials. Sample collection in spring (2010 and 2011) targeted stages 1, 2 and 3 of bud development; which occurred after dormancy release (Cheng et al., 2001). The spring samples targeted aforementioned bud developmental stages not only because each tree peony bud separates into leaves, shoots and flowers, but also because of high bud abortion associated with the plant after these stages (Cheng et al., 2001). The bud samples were harvested using clean secateurs, rinsed in distilled water, and then immediately placed in ice box. The samples were afterwards transported to the laboratory, dipped in liquid nitrogen and stored at $-80{ }^{\circ} \mathrm{C}$ until plant hormone and sugar extractions and analyses.

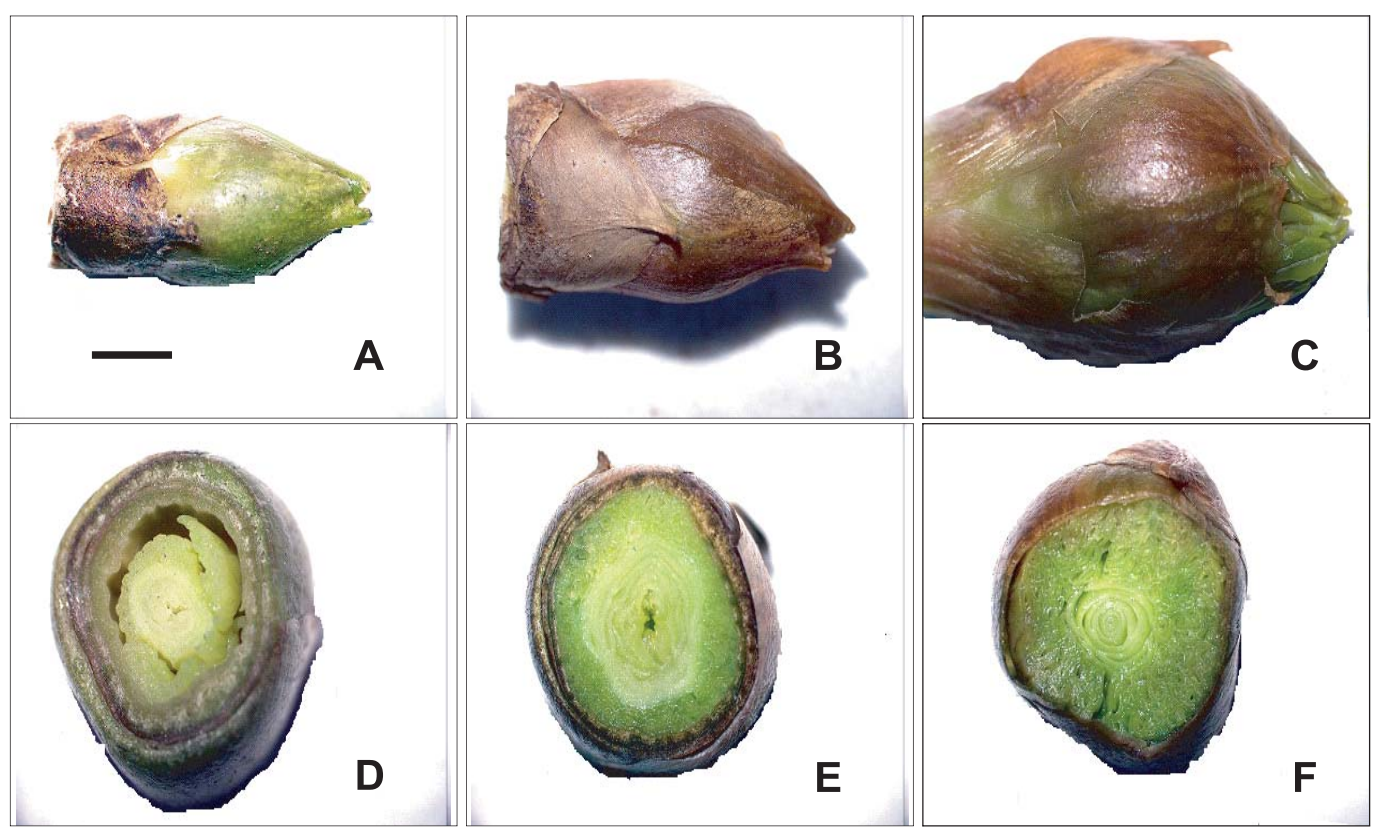

Fig. 1. Photographed sample images of shoot apical bud using computer-aided LEICA DFC500 microscope (scale bar: $0.5 \mathrm{~cm}$ ). A, B \& C show lateral view of the apical bud morphological changes of 'Luoyang Hong' cultivar in autumn, winter and spring, respectively. D, E \& F show vertical view of the internal apical bud structural changes of 'Luoyang Hong' cultivar in autumn, winter and spring, respectively. 'Luoyang Hong' is the most popular cultivar used in flower forcing and is of high ornamental value in China. 
Bud dormancy intensity: The degree of dormancy was indirectly estimated in terms of bud-break response under field conditions. About 120 buds of each cultivar were randomly selected and analyzed for percent bud dormancy release and days to budbreak. The percent of bud dormancy release was calculated as the number of balloon-shaped or swollen buds with green-purple scales.

Bud morphology: Shoot apical buds were randomly harvested for close morphological observation. About 27 buds were randomly selected for this purpose. The images were photographed using computer-aided LEICA DFC500 microscope (Fig. 1).

Hormone extraction, purification and quantification: With slight modifications, the extraction, purification and quantification of endogenous $\mathrm{GA}_{3}$ and $\mathrm{ABA}$ were done using the ELISA technique as described by He (1993) and Yang et al. (2001). Fresh bud samples $(0.5 \mathrm{~g})$ were homogenized in $5 \mathrm{~mL}$ of $80 \%$ (v/v) methanol, containing $1 \mathrm{mmol} \mathrm{L}^{-1}$ butylated hydroxytoluence (BHT) as antioxidant. About $30 \mathrm{mg}$ silicon dioxide and $20 \mathrm{mg}$ polyvinylpolypyrrolidone (PVP) were added to the homogenate to respectively ease grinding and remove phenol. The extracts were incubated overnight at $4{ }^{\circ} \mathrm{C}$, transferred the next morning into $10 \mathrm{~mL}$ test tubes, and centrifuged at $6000 \mathrm{rpm}$ for $20 \mathrm{~min}$. The supernatant was passed through $\mathrm{C}_{18}$ Sep-Pak cartridge (Waters Corp., Milford, MA) and dried in $\mathrm{N}_{2}$. The residues were dissolved in 0.01 mol. $\mathrm{L}^{-1}$ of phosphate buffer saline (PBS) with $\mathrm{pH} 7.5$ for the determination of $\mathrm{GA}_{3}$ and ABA levels. The mouse monoclonal antigens and antibodies against $\mathrm{GA}_{3}, \mathrm{ABA}$ and IgG-horseradish peroxidase used were produced by the Phytohormones Research Institute, China Agricultural University, Beijing, China. The microtitration plates (Nunc) were coated with synthetic $\mathrm{GA}_{3}$ and with ABA-ovalbumin conjugates in $50 \mathrm{mmol} \mathrm{L}^{-1}$ of $\mathrm{NaHCO}_{3}$ buffer solution ( $\mathrm{pH}$ 9.6), and kept overnight at $37^{\circ} \mathrm{C}$. To block nonspecific binding, $10 \mathrm{mg} \mathrm{mL}^{-1}$ of ovalbumin solution was added to each well. After incubation at $37^{\circ} \mathrm{C}$ for $30 \mathrm{~min}$, standard $\mathrm{GA}_{3}$ and ABA samples and antibodies were added and incubated for another $45 \mathrm{~min}$ at $37^{\circ} \mathrm{C}$. Horseradish peroxidase-labelled goat anti-rabbit immunoglobulin was then added to each well and incubated for $1 \mathrm{~h}$ at $37^{\circ} \mathrm{C}$. The buffered enzyme substrate (orthophenylenediamino) was afterwards added and the enzyme reaction conducted in the dark at $37{ }^{\circ} \mathrm{C}$ for $15 \mathrm{~min}$, and again stopped using 3 mol. $\mathrm{L}^{-1} \mathrm{H}_{2} \mathrm{SO}_{4}$. The ELISA Recorder (Model DG-3022 A; Huadong Electron Tube Factory, Shanghai, China) was used to measure the optical density of each well at $\mathrm{A}_{490} \mathrm{~nm}$. Calculations of the enzyme-immunoassay data were performed following Weiler et al. (1981). The results were given as means and \pm SE (standard error) for the three replicates. In this study, percent recovery of each hormone was calculated by adding a known quantity of standard hormone to a split extract. The percent recoveries were $>90 \%$ and the sample extract dilution curves were parallel to the standard curves. This indicates that no nonspecific inhibitors existed in the extracts.

Soluble sugar extraction and analysis: Fresh buds (1.0 g) were ground in $20 \mathrm{~mL}$ distilled water and extracted in water-bath at $80{ }^{\circ} \mathrm{C}$ for $30 \mathrm{~min}$. The suspension was centrifuged for $10 \mathrm{~min}$ at $6000 \mathrm{rpm}$. The supernatant was used to determine the levels of sucrose and R-sugar and the pellets were used to determine the levels of starch. The sucrose and starch were determined using anthrone reagent (Xue and Xia, 1985), with glucose as a standard.
The R-sugar, on the other hand, was colorimetrically determined using dinitrosalicylic acid. The absorbance was then determined using spectrophotometer (TU-1901).

Statistical analysis: The randomized complete block design with three replicates was used in the experiment. A long crop row consisting of 5 treatment plots made up each replication with 12 plants per plot. Each treatment in the experimental unit consisted of three crop rows each $70 \mathrm{~cm}$ between and $45 \mathrm{~cm}$ within row. The distance between each replication was $1 \mathrm{~m}$ apart and 0.5 between each treatment. Statistical analyses were done using the Statistical Package for Social Scientists (SPSS). The mean seasonal values of the hormones and sugars were used in the one-way ANOVA analysis at $P<0.05$ and $n=3$. Based on this, the \pm SE (standard error) were calculated.

\section{Results}

Bud morphology, dormancy and temperature: The average monthly temperature for the period 2009-2010 is plotted in Fig. 2. Before leaf shedding by the plants in autumn, the outer part of apical buds were green in colour and have started enclosing in two or more pairs of bud scales. For this period, the average temperature was $21 / 8{ }^{\circ} \mathrm{C}$ (day/night). During winter, (when average temperature was $3{ }^{\circ} \mathrm{C}$ for the day time and $-7^{\circ} \mathrm{C}$ for the night time), the plants shed all leaves and the terminal buds were completely enclosed in scales. Cellular elongation had ceased as no visible growth of buds was noted during this period, cell division remained active resulting in the development of floral organ and change in the external bud colour from green to brown (Fig. 1B). Also, during this period the shoot and petal structures were neatly packed together distinguished by different colour with wider central pith in the bud (Fig. 1E). As temperatures became warmer in spring (with average day-time temperature of $10^{\circ} \mathrm{C}$ and night-time temperature of $5{ }^{\circ} \mathrm{C}$ ), the buds started to sprout. The buds swelled and the outer scales became fleshy, bright and changed colour from brown to green-purple. The shoot and petals became loosely packed and the central pith disappeared in the buds (Fig. 1 C \& F).

Dormancy intensity of buds: Dormancy intensity of tree peony buds was calculated by determining the percent bud-break and the days to bud-break of the tested cultivars. For 'LH' buds, 100\% of

Table 1 . Percent bud break and number of days to $60 \%$ post-dormancy release in tree peony

\begin{tabular}{ccccc}
\hline Year & Variable & \multicolumn{3}{c}{ Percent bud-break after dormancy release (\%) } \\
\cline { 3 - 5 } & & LH & ZF & NH \\
\hline 2010 March 4 & $<60$ & 0.0 & 0.0 \\
March 9 & 100 & $<60$ & 0.0 \\
March 14 & - & 100 & $<60$ \\
March 18 & - & - & 100 \\
2011 & & & \\
March 2 & $<60$ & 0.0 & 0.0 \\
March 6 & 100 & $<60$ & 0.0 \\
March 11 & - & 100 & $<60$ \\
March 15 & - & - & 100 \\
& \multirow{2}{*}{ Day count } & Average number of days to 60\% dormancy release \\
\cline { 2 - 4 } & & 155.5 & 162.5 & 165 \\
\hline
\end{tabular}




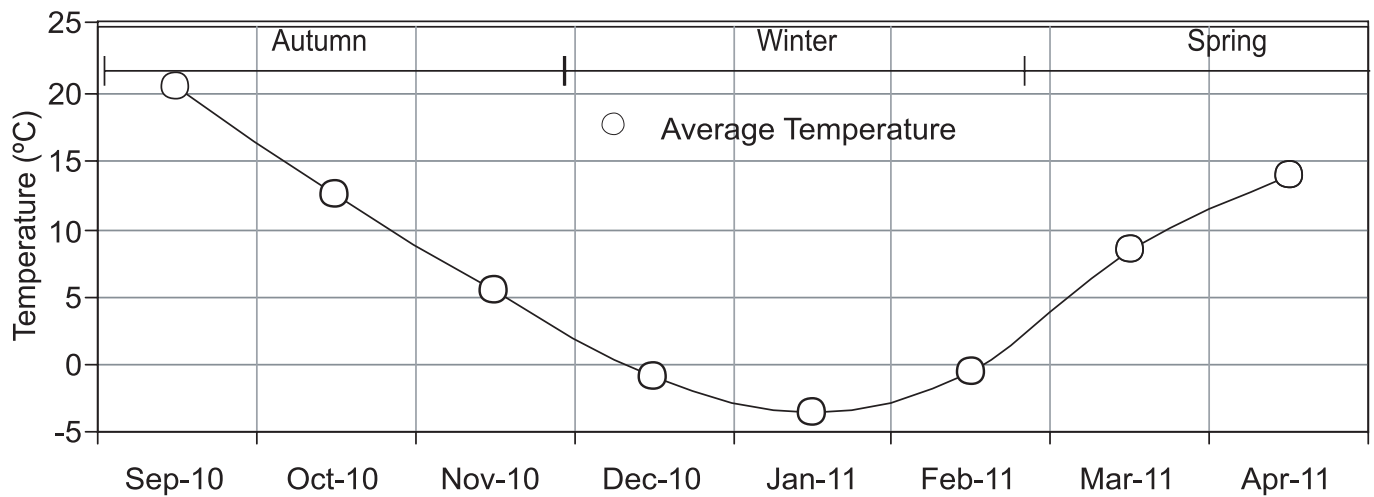

Fig. 2. Average monthly temperature for the autumn, winter and spring seasons of 2009 and 2010 cropping seasons

bud-break was noted on March 9, 2010 and March 6, 2011 for both trials. It was $60 \%$ for 'ZF' and $0.0 \%$ for 'HN' buds. $100 \%$ of bud dormancy release was observed in 'HN' on March 18, 2010 and March 15, 2011, about 10 and 7 days from bud dormancy release in ' $\mathrm{LH}$ ' and 'ZF', respectively (Table 1). To evaluate the difference in bud dormancy intensity in each cultivar, the percent bud-break and days to bud-break (Table 1) were calculated. Based on the calculations, 'HN' had the lowest while ' $\mathrm{LH}$ ' had the highest percent bud-break on March 9, 2010 and March 6, 2011. More number of days were required for $60 \%$ bud-break in ' $\mathrm{HN}$ ' (165 d) than in 'LH' (155 d) and 'ZF' (162.5 d) (Table 1). Field observations showed that bud dormancy release was earlier in 'LH'. March 14, 2010 and March 11, 2011 coincided with stage 3 of bud development after dormancy release in 'LH' (Cheng et al., 2001), which coincided with stages I and II of bud development after dormancy release in ' $\mathrm{HN}$ ' and 'ZF', respectively; indicating that at least 4 days difference in bud development after dormancy release existed among the three cultivars. It indicates that the dates of dormancy release in 'LH', 'ZF' and ' $\mathrm{HN}$ ' were March 2 \& 4, March 6 \& 9 and March 11 \& 14, respectively during 2011 and 2010 .
Hormonal content of bud: In buds of the tested cultivars, different levels of $\mathrm{ABA}$ and $\mathrm{GA}_{3}$ were induced by different temperature regimes with visible patterns of change throughout the year. For all the cultivars, bud ABA levels were generally high in autumn but peaked in winter and then dropped to the lowest levels in spring (Fig. 3A). On the other hand, the levels of endogenous $\mathrm{GA}_{3}$ gradually rose from autumn through winter to spring. When, however, compared with 'ZF' and 'HN', ABA and GA 3 levels in buds of 'LH' responded less to changes in temperature (Fig. 3).

Sugar content of buds: Sugar levels in the tested cultivars have distinct patterns of change with changes in seasonal temperature. The dynamics of starch, sucrose and R-sugar more or less followed that of ABA. It was lower in autumn, peaked in winter and again dropped in spring (Figs. 4A, B \& C). Although the level of sugars generally dropped from winter to spring, it was distinct for sucrose and R-sugar than starch in spring. Among the tested cultivars, starch hydrolysis was more pronounced in ' $\mathrm{LH}$ ' than in 'ZF' and 'HN'. Sucrose was the main accumulated carbohydrate in winter, followed by R-sugar and then starch in the tested cultivars with the 'ZF' and 'HN' accumulating more starch than ' $\mathrm{LH}$ '.
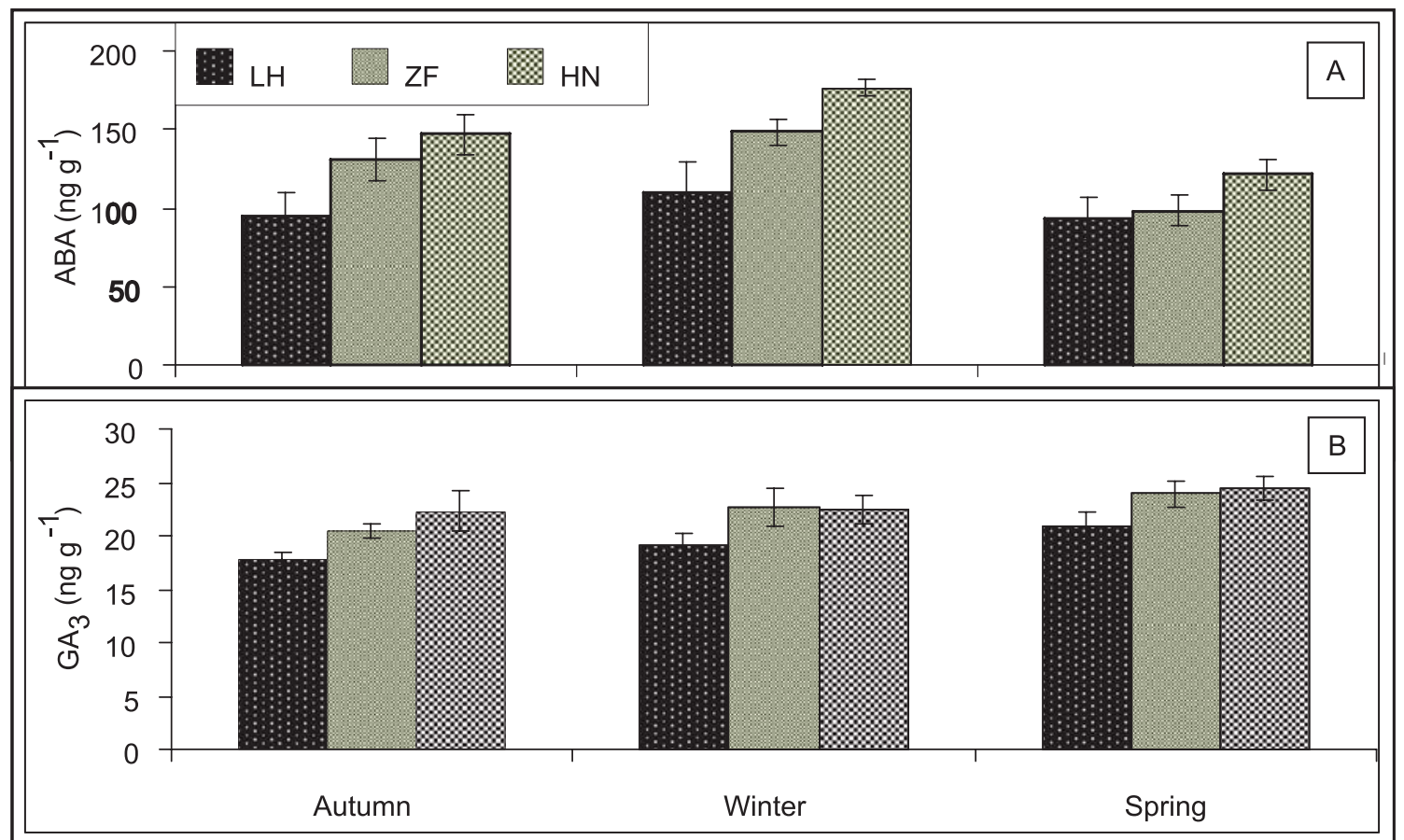

Fig. 3. Average fresh-weight (FW) contents of $\mathrm{ABA}(\mathrm{A})$ and $\mathrm{GA}_{3}(\mathrm{~B})$ hormones in tree peony buds in the autumn, winter and spring of 2009 and 2010 cropping seasons. Bars are the mean \pm SE (standard error) for the three replicate extractions in each season $(n=3)$ 


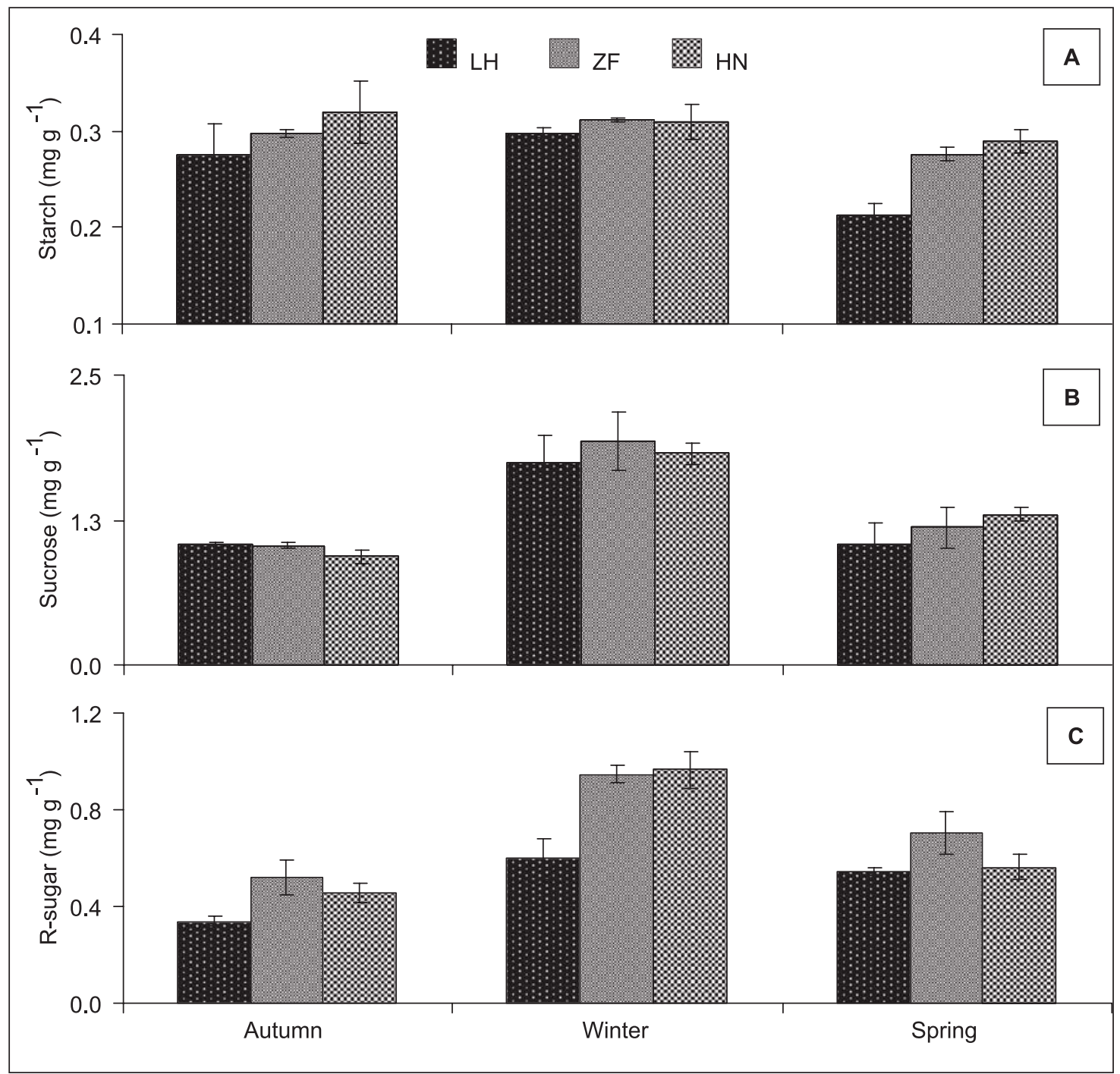

Fig. 4. Average fresh-weight (FW) contents of starch (A), sucrose (B) and R-sugar (C) in tree peony buds in the autumn, winter and spring of 2009 and 2010 cropping seasons. Bars are the mean \pm SE (standard error) for the three replicate extractions in each season $(n=3)$

\section{Discussion}

Bud morphology, dormancy and temperature: Tree peonies have a single bud for leaf, shoot and flower production (mixed bud). The accumulation of low temperature normally enhances chilling requirement required for flower bud growth. The development of tree peony flower buds normally starts in late summer, followed by leaf senescence and bud dormancy. Bud development only resumes after sufficient cold vernalization, followed by blooming in spring (Wang et al., 1998). The distinct events that occurred in winter during dormancy phase include leaf shedding by plants, complete enclosure of buds in scales, change in external bud colour to brown, cessation of visible growth of buds and compacting of floral organs in the buds. The stems and buds eventually became important as site of photoperiod signal reception and low temperature stimuli. In spring during dormancy release stage, the marked changes noted were swelling of buds, change of external bud colour from brown to purplegreen and loosing of the floral organ within buds coupled with the disappearance of the central pith (Fig. 1F).

Relationship among hormone, dormancy and temperature: Dormancy is always closely related with temperature (Junttila et al., 2003; Molmann et al., 2005). Though previous research has demonstrated that photoperiod is a basic environmental stimulus for dormancy induction, hormones also can regulate many other aspects of dormancy such as its timing and intensity. Several studies attributed bud dormancy development in woody species to decreasing temperatures and increasing levels of ABA (Molmann et al., 2005; Rinne et al., 1994; Welling et al., 1997). There are also studies that attribute bud dormancy development to increasing temperatures (Fuchigami et al., 1982; Palonen, 2006). In this study, increasing levels of ABA in buds of the tested tree peony cultivars coincided with increasing low temperature in winter and dormancy development. As the temperature became warmer in spring, the level of ABA substantially decreased. Our results demonstrated that the levels of $\mathrm{ABA}$ and $\mathrm{GA}_{3}$ are closely related to bud dormancy and germination induced by changes in temperature. The increase in ABA levels in winter and then decrease in spring implies that winter temperatures possibly induced ABA accumulation, which in turn resulted in bud dormancy development. Winter temperature could trigger biosynthesis or retard ABA degradation in buds. The finding in this study regarding temperature-ABA relationship in tree peonies is consistent with those in Junttila et al. (2003) and 
Rinne et al. (1994) for Betula spp and Powell (1987) for other woody plants.

The relationship among endogenous GA, bud dormancy and temperature has also been investigated in grapes (Lavee and May, 1997), apples (Qin et al., 2009) and in sweet cherry (Duan et al., 2004). These studies concluded that though low temperature decreases bud $\mathrm{GA}_{3}$ level during bud dormancy period, the level increases during dormancy release with increasing temperatures. Buchheim et al. (1994) also reported positive effect of $\mathrm{GA}_{3}$ on epicotyl dormancy release in cultured herbaceous peony embryos. In the present study, the levels of $\mathrm{GA}_{3}$ increased with increasing temperature during spring in all cultivars. The increase in $\mathrm{GA}_{3}$ level coincided with bud sprout stage, which indicates bud dormancy release in tree peonies. The apparent inverse relationship in the trends between $\mathrm{GA}_{3}$ and $\mathrm{ABA}$ (i.e., $\mathrm{GA}_{3}$ levels increase as ABA levels decrease with increase in temperature) is consistent with what is generally known as antagonistic interaction between $\mathrm{GA}_{3}$ and $\mathrm{ABA}$ with regards to changes in temperature. Previous researches have demonstrated that ABA must drop to a threshold level beyond which GA 3 activity can increase to induce bud dormancy release. An increase in $\mathrm{GA}_{3}$ levels during dormancy release in spring was observed in all tested cultivars, which not only suggest that ABA and $\mathrm{GA}_{3}$ have opposing effects at dormancy release, but also regulate dormancy in the plants. The respective high and low levels of ABA and $\mathrm{GA}_{3}$ possibly inhibits and promotes the production of essential RNA (Alexopoulos et al., 2008) for source-sink translocation of amino acids and sugars (Rashid, 2009), which in turn respectively inhibits and promotes bud growth in tree peonies. The decline in ABA level in spring possibly induced the $\mathrm{GA}_{3}$ biosynthesis resulting in increase in $\mathrm{GA}_{3}$ level. $\mathrm{GA}_{3}$ is normally associated with growth acceleration in plants (Qin et al., 2009).

Trend in sugar, dormancy and temperature: Seasonal variations in the levels of sugars are noted in woody plants. In general, total soluble plant sugars start to rise at the onset of cold acclimation in winter, reach the maximum at full-cold hardiness, and drop at deacclimation in spring (Kozlowski, 1992; Pallardy, 2008; Sahai and Larcher, 1987). Seasonal dynamics of sugars were also noted in this study, in which increase in starch, sucrose and R-sugar levels coincided with decrease in winter temperature and bud dormancy development, and then decreased with increase in spring temperature and bud dormancy release. This implies that enhanced biosynthesis of these elements could be central for cold acclimation and that their levels could regulate bud dormancy development and release in tree peonies.

The rise in sugar levels in winter and fall in spring could be driven not only by temperature changes, but also by bud developmental requirements. The leaves of tree peony dry off and shed during winter. Therefore, minimal amount of sugars is required for bud respiration and maintenance during winter. Any stored reserves of sugars could be used in the following spring to provide energy for the growth of developing shoots (Otzen and Koridon, 1970). Studies have suggested that sucrose accumulates during winter bud dormancy and degrades during spring when bud growth resumes, whereas starch level shows inverse relationships (Miller and Langhans, 1990; Kozlowski, 1992). In this study, similar seasonal change patterns were noted in tree peony bud's sucrose and starch contents — both elements peaked in winter and fell in spring.
Sucrose was the main form of accumulated sugars in the tested cultivars (Fig. 4B), which suggests that despite seasonal and cultivar variations, sucrose is the main source of sugar in tree peonies. It could be mainly derived from the transformation of stored starch to provide energy and carbon skeleton for the synthesis of needed amino acids, lipids and metabolites for plant growth (Kozlowski, 1992; Pallardy, 2008). The hydrolysis of starch was lesser in spring, however, the reverse holds true for sucrose and R-sugar. Even so, starch hydrolysis among the tested cultivars was greater in 'LH' than in 'HN' and 'ZF' (Fig. 4A). This suggests that starch degradation increased sugar levels of ' $\mathrm{LH}$ ', which could also be one of the prime reasons for its earlier bud sprout as sugar is reportedly necessary to sustain flower buds development (Kozlowski, 1992; Pallardy, 2008). Starch accumulated during winter when buds were dormant and then converted into sugar during dormancy release in spring possibly alleviated freeze damage of buds and it is of great importance as it increases the osmotic potential in the cell, thus increasing frost resistance (Krabel et al., 1994). The conversion of starch into sugar coincided with increase in $\mathrm{GA}_{3}$ level indicating that processes leading to the synthesis of amylase are controlled by $\mathrm{GA}_{3}$.

Lesser accumulation of ABA, GA $\mathrm{A}_{3}$ and sugar levels in buds of ' $\mathrm{LH}$ ' followed by ' $\mathrm{ZH}$ ' and ' $\mathrm{HN}$ ', indicate that the response of these internal compositions to changes in temperature was slower in ' $\mathrm{LH}$ ' than in ' $\mathrm{ZF}$ ' and 'HN'. This could be attributed to the genetic differences among the three cultivars. The variations in the ability to accumulate ABA, $\mathrm{GA}_{3}$ and sugars could influence the depth of dormancy in these cultivars, as clearly manifested in their percentage bud-break and days to dormancy release (Table 1). It was noted that changes in internal compositions of the tested cultivars were triggered by temperatures and hence variation in their dormancy releasing time. Compared with ' $\mathrm{ZF}$ ' and ' $\mathrm{HN}$ ', 'LH' which depicted the least accumulation of bud's ABA, $\mathrm{GA}_{3}$ and sugars contents during spring released bud dormancy earlier (155 days) with the highest percentage of bud-break (Table 1), indicating that dormancy intensity was lesser in ' $\mathrm{LH}$ ' and therefore, may require lower cumulative low temperature for fullfilling chilling requirements. Whereas there was about 7 days difference in dormancy release between ' $\mathrm{LH}$ ' and ' $\mathrm{ZF}$ ', it was 10 days between 'LH' and 'HN' (Table 1). In spring, bud sucrose levels in ' $\mathrm{ZF}$ ' and ' $\mathrm{HN}$ ' were slightly higher than in 'LH'. High levels of sucrose could inhibit bud growth (Katovich et al., 1998; Kozlowski, 1992) and this could be another factor for earlier bud germination in ' $\mathrm{LH}$ ' in spring. Accordingly, low levels of ABA and sugars could be essential for bud dormancy release and transformation in tree peonies. The ability of the buds to simultaneously accumulate ABA and sugar while in dormant state may provide a significant adaptive advantage for peonies to survive the erratic climate, particularly in temperate regions. This could be one of the reasons for the geographically widespread of the genus Paeonia in the world.

In conclusion, dormancy in winter is likely to be caused by increase in ABA, starch, sucrose and R-sugar levels in tree peony buds which in turn inhibits the growth of flower structures. While these elements peaked in winter, they fell back to low levels with increasing temperatures in spring. $\mathrm{GA}_{3}$ showed a steady increase from autumn through spring. The results suggest that 
ABA and sugar accumulation inhibit tree peony bud growth, $\mathrm{GA}_{3}$ accumulation, and ABA and sugar content decline facilitate it with temperature as a regulatory factor. Compared with ' $\mathrm{ZF}$ ' and 'HN', ABA, GA 3 and sugar contents in 'LH' were less responsive to seasonal changes in temperature resulting in their less accumulation and earlier dormancy release in ' $\mathrm{LH}$ '. Therefore, to regulate dormancy break in favour of commercial tree peony flower production, regulation of $\mathrm{ABA}, \mathrm{GA}_{3}$ and sugars could be required for timely dormancy release.

\section{Acknowledgment}

This work was funded by the National Science and Technology Support Program of China (2006BAD01A1801), Key Project for Forestry Science Research (2006-40) and the Co-constructive Project of Beijing Education Committee (2009).

\section{References}

Buchheim, J.A.T., L.F. Burkhartand and M.M. Meyer, Jr. 1994. Effect of exogenous gibberellic acid, abscisic acid and benzylaminopurine on epicotyl dormancy of cultured herbaceous peony embryos. Plant Cell, Tissue and Organ Culture, 36: 35-43.

Burke, M.J., L.V. Gusta, H.A. Quamme, C.J. Weiser and P.H. Li, 1976. Freezing and injury in plants. Annual Review of Plant Physiology, 27: 507-528.

Byrne, T.G. and A.H. Halevy, 1986. Forcing herbaceous peonies. Journal of the American society for Horticultural Science, 111: 379-383.

Chen, H.H. and P.H. Li, 1978. Interaction of low temperature, water stress, and short days in the induction of stem frost hardiness in red-osier dogwood. Plant Physiology, 62: 833-835.

Cheng, F.Y., N. Aoki and Z.A. Liu, 2001. Development of forced tree peony and comparative study of pre-chilling effect on Chinese and Japanese cultivars. Journal of the Japanese Society Horticultural Science, 70 (1): 46-53.

Duan, C.G., X.L. Li, D.S. Gao and M. Li, 2004. Studies on regulations of endogenous hormones ABA and GA in sweet cherry flower buds on dormancy. Acta Horticulturae Sinica, 31: 149-154.

He, Z., 1993. Guidance to Experiment on Chemical Control in Crop Plants. Beijing Agricultural University Publishers, Beijing. p. 60-68.

Junttila, O., J. Nilsen and B. Igeland, 2003. Effect of temperature on the induction of bud dormancy in ecotypes of Betula pubescens and Betula pentandra. Scandinavian Journal of Forest Research, 18: 208-217.

Katovich, E.J.S., R.L. Becker, C.C. Sheaffer and J.L. Halgerson, 1998. Seasonal fluctuations of carbohydrate levels in roots and crown of purple loosestrife (Lythrum salicaria). Weed Science, 46: 540-544.

Kozlowski, T.T. 1992. Carbohydrate sources and sinks in woody plants. Bot. Rev., 58: 107-222.

Krabel, D., M. Bodson and W. Eschrich, 1994. Seasonal changes in the cambium of trees. I. Sucrose content in Thuja occidentalis. Botanical Acta, 107: 54-59.

Lavee, S. and P. May, 1997. Dormancy of grapevine buds-facts and speculation. Australian Journal Grape Wine Research, 3: 31-46.

Nowak, J., M. Saniewsky and R.M. Rudnicki, 1974. Studies on the physiology of hyacinth bulbs (Hyacinthus orientalis). I. sugar content and metabolic activities in bulbs exposed to low temperatures. Journal Horticultural Science, 49: 383-390.
Olsen, J.E., O. Junttila and T. Moritz, 1997. Long-day induced bud break in Salix pentandra is associated with transiently elevated levels of $\mathrm{GA}_{1}$ and gradual increase in indole-3-acetic acid. Plant Cell Physiology, 38(5): 536-540.

Otzen, D. and A.H. Koridon, 1970. Seasonal fluctuations of organic food reserves in underground parts of Cirsium arvense (L.) Scop. and Tussilago farfara L. Acta Bot. Neerl., 19: 495-502.

Pallardy, S.G. 2008. Physiology of Woody Plants. Third edition. Elsevier Inc. USA.

Powell, L.E. 1987. The hormonal control of bud and seed dormancy in woody plants. In: Plant Hormones and their Role in Plant Growth and Development. P.J. Davies (eds.). Martinus Nijhoff, Dordrecht. p. 539-552.

Palonen, P. 2006. Vegetative growth, cold acclimation, and dormancy as affected by temperature and photoperiod in six red raspberry (Rubus idaeus L.) cultivars. European Journal of Horticultural Science, 72(4): 1-6.

Pinthus, M.J., M.D. Gale, N.E.J. Appleford and J.R. Lenton, 1989. Effect of temperature on gibberellin (GA) responsiveness and on endogenous GA1 content of tall and dwarf wheat genotypes. Plant Physiology, 90: 854-859.

Qin, D., J.Z. Wang, J.M. Guo and H. Zhai, 2009. The relation between endogenous hormones and late-germination in buds of avrolles apple. Agricultural Science in China, 8(5): 564-571.

Rashid, A. 2009. Molecular Physiology and Biotechnology of Flowering Plants. Alpha Science International Ltd. Oxford, UK.

Rinne, P., H. Tuominen and O. Junttila, 1994. Seasonal changes in bud dormancy in relation to bud morphology, water and starch content, and abscisic acid concentration in adult trees of Betula pubescens. Tree Physiology, 14: 549-561.

Sakai, A. and W. Larcher, 1987. Frost Survival of Plants, Responses and Adaptations to Freezing Stress. Ecological Studies 62. SpringerVerlag, Berlin, Germany.

Thingnaes, E., S. Tore, A. Ernstsen and R. Moe, 2003. Day and night temperature responses in Arabidopsis: Effects on gibberelin and auxin content, cell size, morphology and flowering time. Annals of Botany, 92: 601-612.

Wang, L.Y., K.J. Qin, J.M. Wu and H. Yu, 1998. Chinese Tree Peony. China Forestry Publishing House, Beijing, China.

Weiser, C.J. 1970. Cold resistance and injury in woody plants. Science, 169: 1269-1278.

Weiler, E.W., P.S. Jordan and W. Conrad, 1981. Levels of indol-3-acetic acid in intact and decapitated coleoptiles as determined by a specific and highly sensitive solid-phase enzyme immunoassay. Planta, 153: 561-567.

Welling, A., P. Kaikuranta and P. Rinne, 1997. Photoperiodic induction of dormancy and freezing tolerance in Betula pubescens. Involvement of ABA and dehydrins. Plant Physiology, 100:119-125.

Xue, Y.L. and Z.A. Xia, 1985. The Handbook of Plant Physiology. $1^{\text {st }}$ ed. Shanghai, Shanghai Technology and Science Publishing House Press. 134-135.

Yang, Y.M., C.N. Xu, B.M. Wang and J.Z. Jia, 2001. Effects of plant growth regulators on secondary wall thickening of cotton fibres. Plant Growth Regulation, 35: 233-237.

Received: March, 2012; Revised: January, 2013; Accepted: May, 2013 